\title{
Psychometric evaluation of the MSU CAM health literacy scale
}

\section{Authors: Clarann Weinert, Jean Shreffler-Grant, and Elizabeth Nicholas}

NOTICE: this is the author's version of a work that was accepted for publication in Complementary Therapies in Medicine. Changes resulting from the publishing process, such as peer review, editing, corrections, structural formatting, and other quality control mechanisms may not be reflected in this document. Changes may have been made to this work since it was submitted for publication. A definitive version was subsequently published in Complementary Therapies in Medicine, VOL\# 42, (February 2019), DOI\# 10.1016/j.ctim.2018.11.007.

Weinert, Clarann, Jean Shreffler-Grant, and Elizabeth Nicholas. "Psychometric evaluation of the MSU CAM health literacy scale." Complementary Therapies in Medicine 42 (February 2019): 156-157. DOI:10.1016/j.ctim.2018.11.007.

Made available through Montana State University's ScholarWorks scholarworks.montana.edu 
Title: Psychometric Evaluation of the MSU CAM Health Literacy Scale

Authors:

Clarann Weinert, SC, PhD, RN, FAAN

Professor Emerita (Research Scientist), College of Nursing, Montana State University, Bozeman, MT

Mailing address: 419 North $19^{\text {th }}$ Ave. Bozeman, MT 59718

Email: cweinert@montana.edu

Phone: (406) 5879334

Jean Shreffler-Grant, PhD, RN

Professor, College of Nursing, Montana State University, Bozeman, MT

Mailing address: MSU College of Nursing, Missoula Campus, 32 Campus Drive,

Missoula, MT 59812

Email: jeansh@montana.edu

Elizabeth Nichols, PhD, RN, FAAN

Professor Emerita (Research Scientist), College of Nursing, Montana State University,

Bozeman, MT

Mailing address: 1558 Henry Drive, Laramie, WY 82072

Email: elizabeth59715@gmail.com

Acknowledgements: Funded by a Publication-Driven Research Grant, Montana State University College of Nursing (437266). 


\begin{abstract}
Background/Purpose: The rise in the use of alternative health care approaches (CAM) increases the need for adequate health literacy. The MSU Health Literacy Scale was developed to measure CAM health literacy. Psychometric evaluation of the scale is presented in this article.

Methods: A test retest design was utilized and data from this instrument evaluation research project were used to explore the validity and several forms of reliability of the MSU Health Literacy Scale. The data analysis was based on the scores of 241 individuals at the T1 data collection and the scores of 188 at the T2 administration of the instrument. Results: There were significant correlations between the MSU CAM Health Literacy Scale and Newest Vital Sign $(r=0.330 \mathrm{p}=.000)$ and with a single question health literacy measure $(r=.255(p=.000)$. Cronbach's alphas were adequate for each administration of the scale $(\mathrm{T} 1=.774$ and $\mathrm{T} 2=.754)$. The correlation of scores between the $\mathrm{T} 1$ administration and the T2 administration were significant $(r=.696(\mathrm{p}=.000)$. Conclusions: This psychometric evaluation provides confirmation of convergent validity and stable reliability. The scale can be used in future research and clinical endeavors.
\end{abstract}




\section{Psychometric Evaluation of the MSU CAM Health Literacy Scale}

\section{Introduction}

Adequate health literacy is widely recognized as a necessity in today's health care market place so that consumers can take an active role in decision-making about their health and options for care (Institute of Medicine, 2004). Health literacy is defined as the capacity to obtain, process, communicate, and understand basic health information and services needed to make appropriate health decisions (Koh, Brach, Harris, \& Parchmen, 2013). With increased use of herbal products and dietary supplements, the need for health literacy about complementary and alternative health approaches is also important. The purpose of this research brief is to report the psychometric evaluation of the MSU CAM Health Literacy Scale.

Through a series of studies on the use of CAM among older adults living in sparsely populated areas in the western United States, the need for education to improve CAM health literacy was identified (Nichols, Sullivan, Ide, Shreffler-Grant, \& Weinert, 2005; ShrefflerGrant, Hill, Weinert, Nichols, \& Ide, 2007). Critical to developing a CAM health literacy skill building intervention was the ability to measure CAM health literacy. The available health literacy instruments measured reading and numeracy skills, not the broader range of knowledge and cognitive skills needed for adequate health literacy when making decisions about using selfprescribed CAM. To enable the assessment of the level of CAM health literacy for clinical and/or research purposes a strong, theoretically grounded measure was essential. The MSU CAM Health Literacy conceptual model was developed through a lengthy iterative process of deriving constructs, concepts, and empirical indicators from the literature and the research team's prior work, with review and critique by experts and formed the structure for the development of the MSU CAM Health Literacy Scale (Shreffler-Grant, Nichols., Weinert, \& Ide, 2013; Shreffler- 
Grant, Weinert, \& Nichols, 2014).

\section{Methods}

After this research project was approved by the Montana State University Institutional Review Board for the Protection of Human Subjects, participants were recruited using several strategies. At the time of recruitment participants completed the first questionnaire (T1) containing the MSU CAM Health Literacy Scale, two general health literacy measures, and a short demographic sheet.

Two weeks later the second questionnaire (T2) packet was mailed containing a cover letter, the MSU CAM Health Literacy scale, and an addressed/stamped return envelope. Participants were asked to complete the questionnaire within a week thus providing a test retest window of about 3 weeks.

The MSU CAM Health Literacy Scale is a knowledge-based instrument for use in measuring health literacy in a CAM context. The scale consists of 21 items with initial Cronbach's alphas of .75 to .79. The detailed discussion of the development of the scale, the initial psychometric evaluation, and a copy of the full measure are provided in an earlier article (Shreffler-Grant, Weinert, \& Nichols, 2014).

To assess convergent validity, general health literacy was measured using "The Newest Vital Sign" a six item scale with a reported Cronbach's alpha of $>0.76$ (Weiss et al, 2005). Osborne et al (2007) reported that this scale is a valid and reliable screening tool for health literacy. Additionally, a single general health literacy question, "How confident are you filling out medical forms by yourself?" with five response options ranging from 1 "Not Confident at All" to 5 "Extremely Confident" was included. This single question has been demonstrated to detect inadequate health literacy (Chew, Bradley, \& Boyko, 2004). 


\section{Results}

A total of 251 individuals participated in the T1 data collection resulting in 241 useable questionnaires for analysis. Of those providing useable data $189(76.8 \%)$ were women. The age range was 21 to 95 years with a mean age of 60.5 years. Over 47 percent (47.3\%) were currently married or partnered and $68.8 \%$ had more than a high school education. A total of 193 individuals returned the T2 questionnaire and 188 were useable. Of those who returned useable data, $145(77.1 \%)$ were women with a mean age of 59.9 years.

The possible range of scores on the MSU CAM Health Literacy Scale is 21 to 84. Participants were fairly CAM health literate with a mean score of $70.19(\mathrm{sd}=6.85)$ and a range of scores of 51 to 83 .

The gold standard indicator for reliability is Cronbach's alpha. In this test retest study the alpha was .774 at the T1 administration of the MSU CAM Health Literacy Scale. At the T2 the alpha was .754 .

The correlation of the total scores on the first administration (T1) of the MSU CAM Health Literacy Scale with the scores on the second administration (T2) was $r=.696(p=.000)$. It is commonly held that if the correlation between separate administrations of a measure is high ( 0.70 or higher) then it has good test retest reliability.

To assess convergent validity, the scores on the MSU CAM Health Literacy Scale were compared to scores on the Newest Vital Sign and the single question health literacy measure. The possible range of scores on the Newest Vital Sign is 1 to 6 with a mean score in this study of $4.72(\mathrm{sd}=1.92)$ and an alpha of .876. On the single question health literacy measure, $80.9 \%$ indicated that they were either extremely or quite confident filling out medical forms by 
themselves. The correlation between the MSU CAM Health Literacy Scale and the Newest Vital Sign was $r=0.330(p=.000)$ and with the single question health literacy measure the correlation was $r=.255(\mathrm{p}=.000)$.

The association of demographic variables with the MSU CAM Health Literacy Scale was also examined. The scale was correlated significantly with education $r=.315(\mathrm{p}=.000)$ and with being a user of CAM r=.161 $(\mathrm{p}=.013)$.

\section{Discussion}

The results of this psychometric assessment of the MSU CAM Health Literacy Scale added credibility to the scale. While this is encouraging there are limitations concerning validity assessment. As the scale was designed to measure CAM health literacy, a construct that had not been measured prior to this scale, there is no gold standard to use as a solid comparison for assessing validity. The validity assessment results in this current study were statistically significant but the correlations were modest. The available general health literacy measures are limited in scope to the assessment of basic reading comprehension and numeracy skills (Institute of Medicine, 2004). Given the nature and focus of the available general health literacy measures these results lend credence that the CAM scale is consistent with the domain of health literacy. Health literacy involves the capacity to obtain, process, communicate, and understand basic health information and services needed to make appropriate health decisions. The complexity of this capacity requires more knowledge and skills than basic reading and math, and is a challenging construct to measure accurately.

The MSU CAM Health Literacy Scale has now undergone extensive reliability evaluation. Over previous studies and this study the Chronbach's alpha has been near or above .70 , indicating adequate reliability. In the process of developing a solid measurement instrument 
it is critical that ongoing psychometric evaluation, using a variety of techniques, be conducted. This test retest study is part of that ongoing evaluation of the MSU CAM Health Literacy Scale and the results provide strong additional confirmation of the scale reliability..

The development and initial use of the scale was with older rural adults. In this current study the participants' ages ranged from 21 to 95 . No statistically significant association between age and scale score was demonstrated. As might be anticipated there was a statistically significant positive relationship demonstrated between CAM health literacy and education and CAM use. In general, individuals with more education are likely to have higher levels of health literacy. Education level has sometimes been used as a proxy for health literacy, although it is known to be an imprecise measure.

Complementary and alternative medicine is widely used, often self-prescribed, and taken based on the recommendations of family, friends, or media advertisements (Institute of Medicine, 2005). Thus, having the capability of assessing the level of CAM health literacy is critical in both research and clinical endeavors. With the additional validity and reliability assessments reported here the MSU CAM Health Literacy Scale is now ready for use in both arenas. The investigators encourage the use of the MSU CAM Health Literacy Scale and welcome feedback so as to allow for continued monitoring of the psychometric properties and to enable refinement of the scale as needed. The MSU CAM Health Literacy Scale and scoring instructions are available in a prior publication (Shreffler-Grant, J., Weinert, C., \& Nichols, E, 2014).

It is anticipated that the scale can have important practice applications as a screening tool for individuals who may use CAM and are at risk for limitations in health literacy about CAM. Should the assessment reveal a limitation, CAM health literacy education can be incorporated 
into the plan of care. Enhancing general and CAM health literacy will enable individuals to make more reasoned and informed health decisions and help avoid harm for those considering the use of CAM. 


\section{References}

Chew, L., Bradley, K., \& Boyko, E. (2004). Brief questions to identify patients with inadequate health literacy. Family Medicine, 36(8), 588-594.

Institute of Medicine. (2004). Health literacy: A prescription to end confusion. Washington, DC: National Academies Press.

Institute of Medicine. (2005). Complementary and alternative medicine in the United States, Washington, DC: National Academies Press.

Koh, H., Brach, C., Harris, L., \&. Parchman, M. (2013). A proposed 'health literate care model' would constitute a systems approach to improving patients' engagement in care. Health Affairs, 32(2), 357-367.

Nichols, E., Sullivan, T., Ide, B., Shreffler-Grant, J. \& Weinert, C. (2005). Health care choices: Complementary therapy, chronic illness, and older rural dwellers. Journal of Holistic Nursing, 23(4), 381-394. doi: 10.1177/0898010105281088

Osborn, C., Weiss, B., Davis, T., Skripkauskas, S., Rodrique, C., Bass, P., \& Wolf, M. (2007). Measuring adult literacy in health care: Performance of the newest vital sign. American Journal of Health Behavior, 31, S36-46.

Shreffler-Grant, S., Hill, W., Weinert, C., Nichols, E., \& Ide, B. (2007). Complementary therapy and older rural women: Who uses and who does not? Nursing Research, 56, 28-33. Doi: 10.1097/00006199-200701000-00004

Shreffler-Grant, J., Nichols, E., Weinert, C., \& Ide, B. (2013). The Montana State University Conceptual Model of Complementary and Alternative Medicine Health Literacy. Journal of Health Communication, 0, 1-8. doi: 10.1080/10810730.2013.778365. 
Shreffler-Grant, J., Weinert, C., Nichols, E., \& Ide, B. (2005). Complementary therapy use among older rural adults. Public Health Nursing, 22, 323-331. doi: 10.1111/j.07371209.2005.220407.x.

Shreffler-Grant, J., Weinert, C., \& Nichols, E. (2014). Instrument to measure health literacy about complementary and alternative medicine. Journal of Nursing Measurement, 22(3), 489-499. Doi: 10.1891/1061-3749.22.3.489

Weiss, B., Mays, M., Martz, W., Castro, K., DeWalt, D., Pignone, M., Mockbee, J., \& Halt, F. (2005). Quick assessment of literacy in primary care: The newest vital sign. Annals of Family Medicine, 3(6), 514-522. Doi: 10.1370/afm.405 